\title{
Studying the dark matter annihilation in galaxy clusters through radio and gravitational lensing measures
}

\section{P. Marchegiani*}

School of Physics, University of the Witwatersrand, Johannesburg, Wits 2050, South Africa

E-mail: Paolo.Marchegiani@wits.ac.za

\section{S. Colafrancesco}

School of Physics, University of the Witwatersrand, Johannesburg, Wits 2050, South Africa

E-mail: Sergio.Colafrancescodwits.ac.za

Galaxy clusters are expected to be dominated by a component of Dark Matter (DM) of unknown nature. The annihilation of DM particles in a galaxy cluster can produce relativistic electrons and gamma rays, that can be used to test the properties of the DM particles and of the cluster itself. Gravitational lensing measures provide the spatial DM distribution in a cluster, and can be used to build detailed models to estimate the contribution of DM-produced electrons to the non-thermal diffuse emission observed in some galaxy clusters. Therefore putting together the information derived from radio and gravitational lensing measures in galaxy clusters can provide important information about the properties of DM and the physics of clusters.

We apply this technique to two galaxy clusters where radio and gravitational lensing measurements are available. In the Bullet cluster a contribution of the DM to the diffuse radio emission is possible because of the spectral changes visible in the overall spectrum, that suggest that more than one component can be present; to better constrain this possibility information on the spectrum of the diffuse radio emission in smaller regions of the cluster is necessary. In the Coma cluster the distribution of the DM sub-halos closely resembles the radio halo surface brightness shape, and the overall radio halo spectrum is well reproduced by a DM model for the observed properties of the magnetic field, without violating the gamma ray upper limits in the cluster; however, there are open issues, like the values of the annihilation cross section and of the substructures boosting factor.

On the basis of these results, we conclude that the combination of radio and gravitational lensing studies of galaxy clusters appears to be a very promising way to obtain information about the physics of galaxy clusters and the DM properties. Present results suggest that a contribution from DM to the total diffuse radio emission can be important, but require to study in detail the spectrum of the diffuse radio emission obtained in smaller regions of the clusters, and to have good contraints on the components of baryonic origin. The application of this technique to a higher number of clusters will be important to obtain better information and solve some open issues.

5th Annual Conference on High Energy Astrophysics in Southern Africa

4-6 October, 2017

University of the Witwatersrand (Wits), South Africa

* Speaker. 


\section{Introduction}

Galaxy clusters are the largest gravitationally bound structures in the Universe, and are dominated by a component of Dark Matter (DM) of unknown nature. The annihilation of DM particles in a galaxy cluster can produce relativistic electrons and gamma rays, whose detection can be used to test the properties of the DM particles and of the cluster itself [1]. However, other processes in galaxy clusters can produce diffuse non-thermal emission in several spectral bands, like radio, $\mathrm{X}$-rays, and gamma rays [2], making difficult to distinguish the emission of baryonic origin from the one originated by DM annihilation.

Previous studies [1,3] have shown that the diffuse radio emission due to DM-produced electrons interacting with the intra-cluster magnetic field can have intensity and spectrum similar to the radio halos observed in several clusters, whereas the expected spatial distribution of the radio surface brightness results to be different from the observed one. In these studies, the DM spatial distribution was modeled as a single spherically symmetric halo centered on the X-ray center of the cluster. However, gravitational lensing studies of the DM spatial distribution in galaxy clusters have shown that this assumption is not always accurate; in fact in the Coma cluster the DM is structured in many sub-halos [4], and in the Bullet cluster the DM is concentrated in two main halos located in different positions with respect to the X-ray emission of the cluster [5].

Therefore, gravitational lensing measures provide information on the DM distribution in clusters that need to be taken into account in accurate DM models. In this way, it is possible to obtain more reliable information about the regions of the cluster where the DM can give a relevant contribution to the observed non-thermal emission, and to distinguish the DM from the baryonic emission combining this information with the radio observations.

In this paper, we report the results obtained in the cases of the Bullet cluster [6] and the Coma cluster [7], discuss and compare the results obtained in the two clusters, and discuss new possible applications of this technique.

\section{The DM model}

Gravitational lensing measurements provide the position and the mass of DM halos inside a galaxy cluster. From the mass of a halo $M_{\text {halo }}$ it is possible to derive the properties of the DM spatial distribution inside the halo $[8,9]$. The profile of DM density is written as a Navarro Frenk White profile:

$$
\rho(r)=\frac{\rho_{s}}{\left(\frac{r}{r_{s}}\right)\left(1+\frac{r}{r_{s}}\right)^{2}},
$$

where the scale radius $r_{s}$ of the DM density profile can be derived from the the values of the concentration parameter, $c_{v i r}=R_{v i r} / r_{s}$, and the virial radius $R_{v i r}$. The former can be derived from a fit to the results of cosmological simulations $[8,10]$ :

$$
c_{v i r}=\frac{9}{1+z}\left(\frac{M_{\text {halo }}}{1.3 \times 10^{13} h^{-1} M_{\odot}}\right)^{-0.13}
$$


where $z$ is the cluster redshift and $h$ is the Hubble constant in units of $100 \mathrm{~km} \mathrm{~s}^{-1} \mathrm{Mpc}^{-1}$, while the virial radius is given by:

$$
R_{v i r}^{3}=\frac{M_{\text {halo }}}{\frac{4}{3} \pi \Delta_{c} \rho_{\text {crit }}},
$$

where we assume $\Delta_{c}=100$, and where $\rho_{c r i t}=2.7755 \times 10^{2} h^{2} M_{\odot} \mathrm{kpc}^{-3}$ is the critical density of the universe. The characteristic density $\rho_{s}$ is obtained from the relation:

$$
\frac{\rho_{s}}{\rho_{c r i t}}=\frac{\Delta_{c}}{3} \frac{c_{v i r}^{3}}{\ln \left(1+c_{v i r}\right)-\frac{c_{v i r}}{1+c_{v i r}}} .
$$

Assuming that the DM particle is a Weakly Interacting Massive Particle (WIMP) with mass $M_{\chi}$, the WIMP pair density is $\mathscr{N}_{\chi}(r)=(\rho(r))^{2} /\left(2 M_{\chi}^{2}\right)$, and the production rate of electrons and gamma rays is given by [1]:

$$
Q_{i}(E, r)=\mathscr{B}\langle\sigma v\rangle \sum_{f} \frac{d N_{i}^{f}}{d E} B_{f} \mathscr{N}_{\chi}(r),
$$

where $i$ is the index referring to the output product (i.e. electrons/positrons or photons), $\langle\sigma v\rangle$ is the thermally-averaged WIMP annihilation cross-section, the index $f$ labels annihilation final states with branching ratios $B_{f}$, the production spectra $\left(d N_{i}^{f}\right) /(d E)$ can be calculated using the DarkSusy package [11], and $\mathscr{B}$ is a multiplicative boosting factor produced by the effect of smaller DM substructures [12, 7]. In the following we will use the neutralino as a candidate WIMP, but the results can be valid for a generic WIMP having similar properties.

By the effect of energy losses and spatial diffusion in the magnetized plasma of the cluster, the electrons produced in DM annihilation reach the equilibrium according to the diffusion equation [1]:

$$
\frac{\partial}{\partial t} \frac{d n_{e}}{d E}=\nabla\left(D(E, \mathbf{r}) \nabla \frac{d n_{e}}{d E}\right)+\frac{\partial}{\partial E}\left(b(E, \mathbf{r}) \frac{d n_{e}}{d E}\right)+Q_{e}(E, \mathbf{r}),
$$

where $\left(d n_{e}\right) /(d E)$ is the electron spectrum, $D(E, \mathbf{r})$ is the spatial diffusion function, $b(E, \mathbf{r})$ is the energy-loss function and $Q_{e}(E, \mathbf{r})$ is the electron source function. The equilibrium solution in the case of spherical symmetry and assuming that the energy losses and diffusion terms do not have spatial dependence is of the form:

$$
\frac{d n_{e}}{d E}(E, r)=\frac{1}{b(E)} \int_{E}^{M_{\chi}} d E^{\prime} G\left(E, E^{\prime}, r\right) Q_{e}\left(E^{\prime}, r\right)
$$

where $G\left(E, E^{\prime}, r\right)$ is the appropriate Green's function [1].

\section{The case of the Bullet cluster}

In the Bullet cluster gravitational lensing measures show two main halos located in different positions with respect to the X-rays ones; these observations have been interpreted as due to a separation between baryonic matter and DM as a consequence of a merging event $[5,13]$. The radio halo observed in this cluster has a complex shape, extending around the two X-ray peaks, and also around the DM Eastern (DME) peak [14]. A suitable model to study the radio halo in this cluster therefore can be built using the combination of two baryonic models located in the two 
$\mathrm{X}$-ray peaks, and one DM model in the DME peak [6]. In the following analysis, the properties of the baryonic regions are taken from X-rays $[15,16,17]$, and the ones of the DM regions are taken from gravitational lensing analysis $[5,13]$.

The Main Subcluster (MS) is located where the X-ray emission has its main peak [17] in a very hot region, where also the radio brightness maps show a peak [14]. The radio spectrum of this region has a spectral index of $\alpha_{R} \sim 1.4$ [18]. This spectrum can be tentatively described by using a Warming Rays (WR) model [19], where it is assumed that the structure of the temperature observed in the cluster is given by the equilibrium of the heating provided by cosmic ray protons and the cooling of the Intra Cluster Medium (ICM) by thermal bremsstrahlung, and that the radio emission is due to the secondary electrons produced by hadronic interactions of the cosmic ray protons with the ICM nuclei. By normalizing the amount of cosmic ray protons in this way, it is obtained that their best-fit spatial distribution is proportional to the thermal one, their energy spectrum is a power law with spectral index $s_{p}=2.7$, their pressure is of the order of $42 \%$ of the thermal one, and the required magnetic field intensity is of the order of $7.5 \mu \mathrm{G}$ [6] (see Fig.1, left panel). Alternatively, the radio emission can be produced by primary electrons that are responsible of the non-thermal Sunyaev-Zel'dovich effect possibly observed in the Bullet cluster if their normalized minimum momentum $p=\beta \gamma$ is of the order of 1 [20], for a magnetic field of the order of $8 \mu \mathrm{G}$ [6]. In both cases the radio emission in this region can be explained with a baryonic model, with comparable values of the required magnetic field.
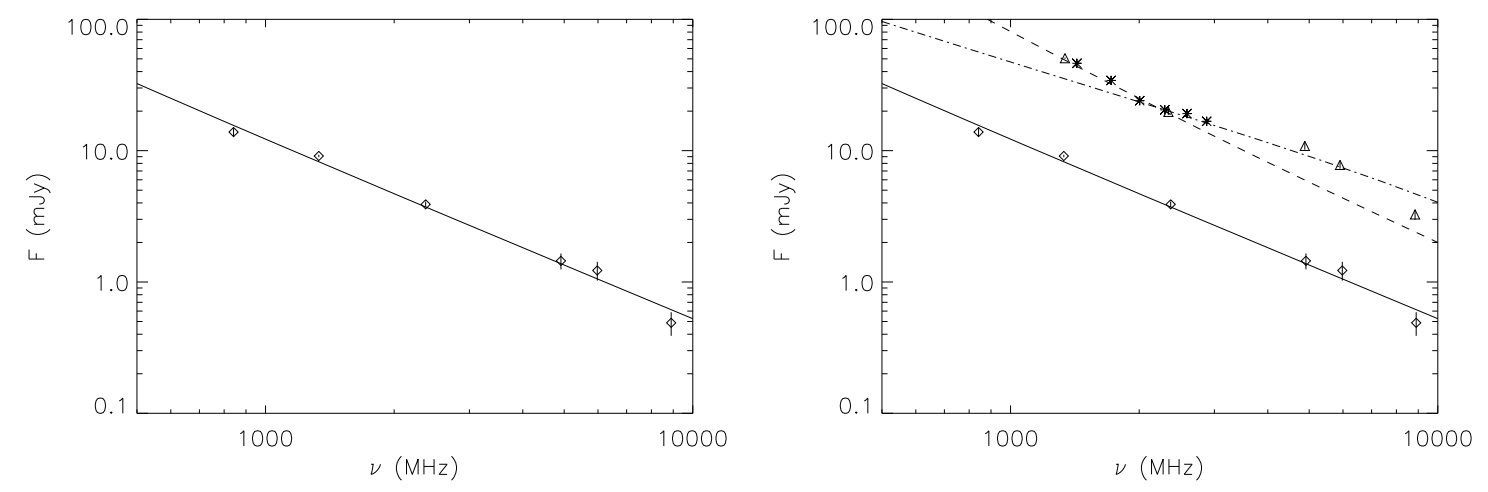

Figure 1: Left Panel: the radio emission in the region of the MS of the Bullet cluster fitted with a WR model with $s_{p}=2.7$ and $B=7.5 \mu \mathrm{G}$. The data shown in this plot correspond to the central region of the cluster as given by Liang et al. [18]. Right Panel: the radio emission in the different regions of the Bullet cluster with: a WR model with $s_{p}=2.7$ and $B=7.5 \mu \mathrm{G}$ in the MS (solid line), a WR model with $s_{p}=2.9$ and $B=60 \mu \mathrm{G}$ in the BS (dashed line), and a DM model with $M_{\chi}=500 \mathrm{GeV}$, composition $W^{+} W^{-}, B=0.015$ $\mu \mathrm{G}$, and $\langle\sigma v\rangle=8.1 \times 10^{-17} \mathrm{~cm}^{3} \mathrm{~s}^{-1}$ in the DME region. Data are taken from region \# 3 of Liang et al. [18] (diamonds), from region \# 2 of Liang et al. [18] (triangles) and from the smaller region of Shimwell et al. [14] (asterisks). Figures from [6].

Unfortunately, in literature there are not available radio spectra integrated in the regions around the Bullet Subcluster (BS) region and the DME peak separately, but only spectra integrated in a wide area including both these regions and the MS. The data sets relative to this wide region $[18,14]$ show a radio halo spectrum with a power-law shape with spectral index $\alpha_{R} \sim 1.6$ up to a frequency 
of $v \sim 2.3 \mathrm{GHz}$, a flattening between 2.3 and $5 \mathrm{GHz}$, and a further steepening at $v \gtrsim 5 \mathrm{GHz}$. This complex spectral shape might suggest that the total spectrum is due to the superposition of different components, one of which can be the BS, and a second flatter component that dominates the overall spectrum at $2.3 \lesssim v \lesssim 5 \mathrm{GHz}$, and steepens at higher frequencies. In Fig.1 (right panel) we show a combination of the previously described WR model in the MS, a second WR model in the BS (where the required pressure ratio between non-thermal and thermal components is $67 \%$ ), and a DM model with $M_{\chi}=500 \mathrm{GeV}$, composition $W^{+} W^{-}$, and magnetic field $B=0.015 \mu \mathrm{G}$ in the DME region, that results to be the best case among a variety of DM models with different mass and composition we considered. This combination of models appears quite good in fitting the data, excluding the point at $8.8 \mathrm{GHz}$ that is overestimated. However, the magnetic field values required by this model are too high in the BS and too low in the DME region compared to galaxy clusters typical values [2], and the required annihilation cross section is higher by a factor of $10^{8}$ compared to the order of magnitude of upper limits found in galaxy clusters with Fermi-LAT measures [21].

We note that an incorrect sources subtraction, which is a delicate operation for extended sources, can alter the estimate of the diffuse radio halo spectrum. Searching for sources inside the radio halo region of the Bullet cluster, we found that the source labeled with A by Liang et al. [18] and with L by Shimwell et al. [14], at coordinates (J2000) RA 06:58:37.9 and Dec -55:57:25, is the only one inside this field with a relatively high flux $(\sim 20 \mathrm{mJy}$ at $1.3 \mathrm{GHz})$, and slightly extended compared with the ATCA resolution of 2.7" [14]. It is possible that the procedure of the flux removal of this source can induce some errors in the estimate of the residual flux attributed to the halo. This source is located very close to the DME peak, and between two galaxies with B magnitude of 20.3 and 21.0 [22]; therefore the radio emission of this source can be produced by one of these galaxies or by an interaction between them, or can be actually the peak of the DM emission. In this case the DM model in the DME region should take in account the flux produced by this source. This is done in Fig.2 (left panel), where the DME emission is normalized to the $\mathrm{A}(\mathrm{L})$ flux spectrum; in this case the baryonic model in the BS requires a flatter protons spectral index compared to the previous case, and a reduced value of the magnetic field (but still high, of the order of $27 \mu \mathrm{G}$ ) in order to reproduce the residual emission. In the figure it is shown also the expected radio emission from the DMW region (assuming the same magnetic field as in the DME region), that instead is not observed.

In Fig.2 (right panel) we show the calculated surface brightness map at $1.5 \mathrm{GHz}$ produced by the same four components as in the left panel, centered on the corresponding positions of the map. This map can be compared to the observed radio map reported in fig.5 of Shimwell et al. [14]. In the simulated map the DM emissions have narrow and intense peaks, but also extended residual emissions; therefore, it is possible that the DM peaks in previous papers have been interpreted as point-like (or slightly extended) sources, while the residual halos have been included in the diffuse cluster emission. We also note that the emission produced in the BS has a limited spatial extension, that does not explain the extended emission observed on the north and on the south of this region. Therefore, to reproduce the full size of the observed radio emission, it is probably necessary to consider the emission produced in a wider region surrounding the BS, or the contributions coming from other sources, like the galaxies halos. 

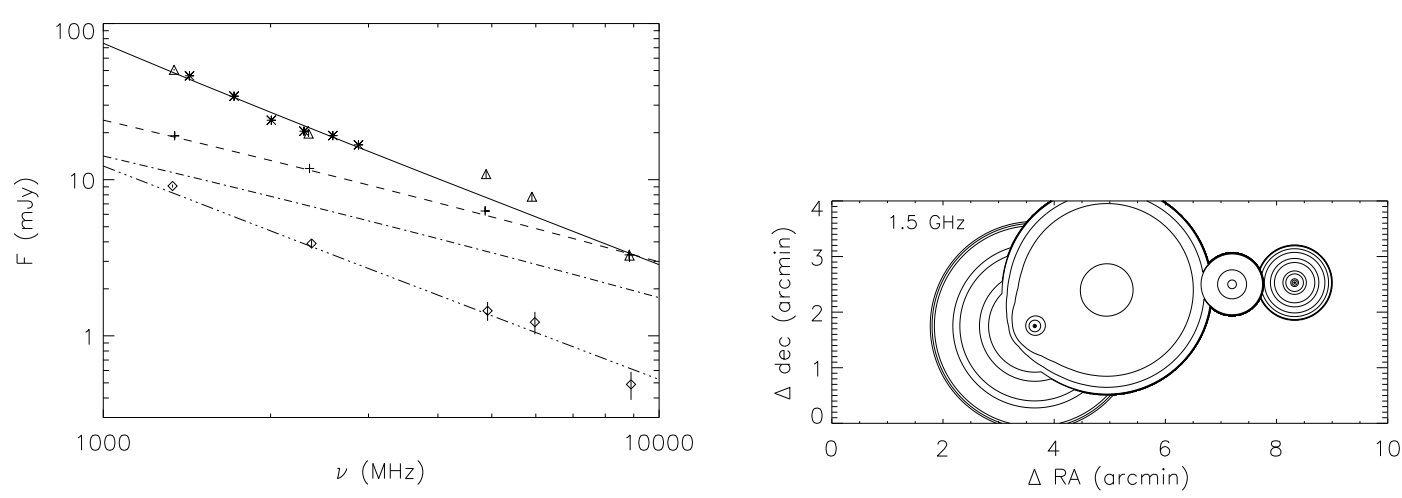

Figure 2: Left Panel: radio emission from the different regions in the Bullet cluster with a WR model with $s_{p}=2.7$ and $B=27 \mu \mathrm{G}$ in the BS (solid line), two DM models with $M_{\chi}=500 \mathrm{GeV}$, composition $W^{+} W^{-}$, $B=10 \mu \mathrm{G}$, and $\langle\sigma v\rangle=4.3 \times 10^{-22} \mathrm{~cm}^{3} \mathrm{~s}^{-1}$ in the DME region (dashed line) and in the DMW region (dotdashed line), and a WR model with $s_{p}=2.7$ and $B=7.5 \mu \mathrm{G}$ in the MS (three dots-dashed line). Data are from region \# 2 of Liang et al. [18] (triangles), from the smaller region of Shimwell et al. [14] (asterisks), from radio source $\mathrm{A}(\mathrm{L})$ from Liang et al. [18] (crosses), and region \# 3 of Liang et al. [18] (diamonds). Right Panel: Radio map of the Bullet cluster at $1.5 \mathrm{GHz}$ simulated using the same models as in the left panel, centered on the corresponding positions on the map. On the axes are the values of the coordinates differences (in arcmin) w.r.t. the origin, fixed in the point RA 06:58:50 and DEC -55:59:00 (J2000). Contour levels correspond to: $\left(1 \times 10^{-6}, 5 \times 10^{-6}, 1 \times 10^{-5}, 5 \times 10^{-5}, 1 \times 10^{-4}, 5 \times 10^{-4}, 1 \times 10^{-3}, 5 \times 10^{-3}\right.$, $1 \times 10^{-2}, 5 \times 10^{-2}, 1 \times 10^{-1}, 5 \times 10^{-1}, 1,5,10,50$, and 100) Jy $\operatorname{arcmin}^{-2}$. Figures from [6].

\section{The case of the Coma cluster}

Gravitational lensing observations in the Coma cluster [4] show that the DM is structured in many sub-halos; interestingly, Brown \& Rudnick [23] noted that the shape of the Coma radio halo morphology is more similar to the distribution of the DM rather than to the X-ray brightness map. In the region centered on the Coma center having a radius of $30 \mathrm{arcmin}$, roughly corresponding to the radius of the Coma radio halo [24], it is possible to identify the position and the mass of 15 sub-halos and of the main halo, having a mass of $1.24 \times 10^{15} \mathrm{M}_{\odot}$ [4].

The equilibrium spectrum of DM-produced electrons is calculated in the main halo and in each sub-halo, considering the effect of the diffusion. The resulting synchrotron emission is calculated using the magnetic field derived from Faraday Rotation measures in the cluster, $B(r)=$ $B_{0}\left(n_{t h}(r) / n_{t h}(0)\right)^{\delta}$ with $\delta=0.5$ and $B_{0}=4.7 \mu \mathrm{G}$ [25], where the thermal gas profile is derived from X-ray measures [26]. Since the size of the DM sub-halos is generally small compared to the dimension of the cluster, we assume that the magnetic field and the gas density are constant within each sub-halo, with the values calculated at the center of the sub-halo.

We assume for the DM particle a neutralino with the compositions and masses corresponding to the models that best fit the Galactic center gamma ray excess according to Abazajian \& Keeley [27]: these are the case with $M_{\chi}=9 \mathrm{GeV}$ and composition $\tau^{+} \tau^{-}$, and the case with $M_{\chi}=43 \mathrm{GeV}$ and composition $b \bar{b}$. Once the neutralino mass and composition are assumed, the only quantity that remains as a free parameter for fitting the data is the normalization, given by the DM annihilation 
cross section $\langle\sigma v\rangle$, eventually multiplied by the substructures boost factor $\mathscr{B}$.

Between the two models considered, the one with mass $9 \mathrm{GeV}$ is found to better fit the shape of the radio halo spectrum of Coma, including the steepening at high frequencies [28]: in Fig.3 (left panel) we show the flux produced from the main halo, the sub-halos, and their sum, and compare with the observed spectrum. The sub-halos give a contribution to the total flux of the order of 5-20\% depending on the DM model. The required values of the normalization of the DM model for the 9 and $43 \mathrm{GeV}$ cases are $\mathscr{B} \times\langle\sigma v\rangle=6 \times 10^{-25} \mathrm{~cm}^{3} \mathrm{~s}^{-1}$ and $4 \times 10^{-24} \mathrm{~cm}^{3} \mathrm{~s}^{-1}$ respectively. Comparing these numbers with the best fit values for the annihilation cross section found in the Galactic center [27], we found that the required value of the substructures boosting factor is of the order of 30-50 for the two models with 9 and $43 \mathrm{GeV}$ mass, that is reasonable for galaxy clusters $[3,29,7]$. If instead the values of the cross section are fixed to the upper limits found in Dwarf Galaxies studies with Fermi-LAT [30] or in CMB studies with Planck [31], we found that the boosting factor should take values of the order of 150-300, that are more difficult but not impossible to have in galaxy clusters $[32,7]$.

We also found that the gamma ray emission produced in this model is not in excess compared to the Fermi-LAT upper limits (see Fig.3, right panel). This is very interesting, because the present gamma ray upper limits put strong constraints on the level of particle acceleration in galaxy clusters, and severely challenge many of the baryonic models presently used to explain the diffuse radio emission in galaxy clusters [33]. At present, DM models seem to not be affected by this problem.
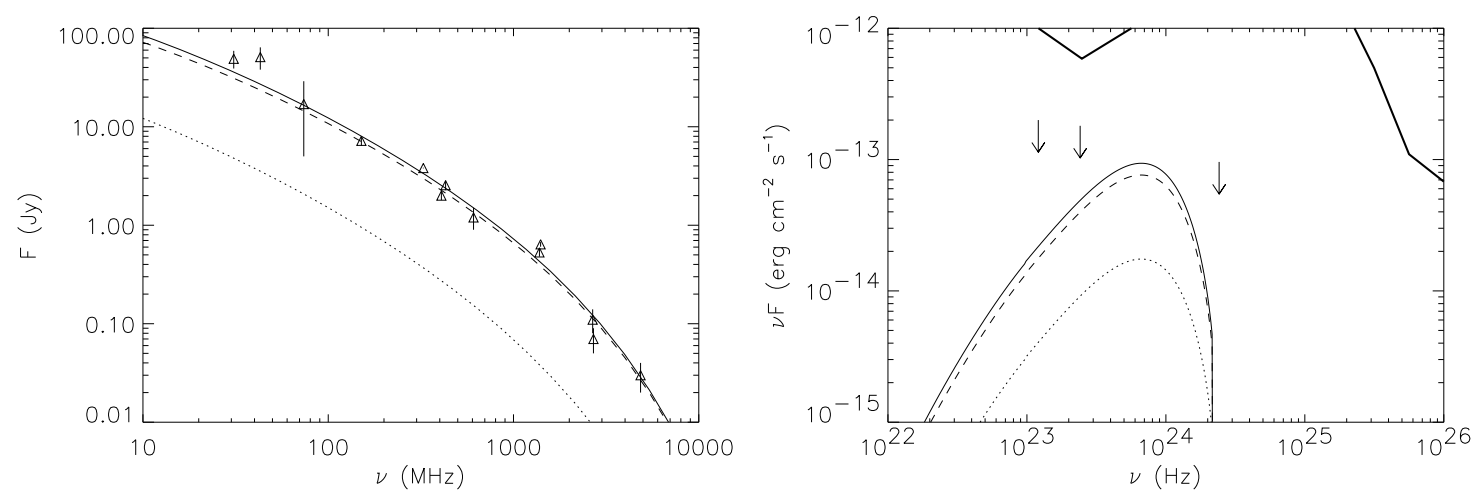

Figure 3: Left Panel: radio flux produced in Coma from the DM halos for a neutralino with $M_{\chi}=9 \mathrm{GeV}$, composition $\tau^{+} \tau^{-}$and $\mathscr{B} \times\langle\sigma v\rangle=6 \times 10^{-25} \mathrm{~cm}^{3} \mathrm{~s}^{-1}$. With the solid line it is shown the total emission, with the dashed line the contribution from the main halo and with the dotted line the contribution from the sub-halos. Data are from Thierbach et al. [28] and references therein. Right panel: gamma ray flux produced in Coma from the DM halos for the same model as in the left panel. The solid line shows the total emission, the dashed line shows the contribution from the main halo and the dotted line shows the contribution from the DM sub-halos. Fermi-LAT upper limits are from [21]. We also plot the expected sensitivities of ASTROGAM for an effective exposure of $1 \mathrm{yr}$ (from http://astrogam.iaps.inaf.it/scientific_instrument.html) and CTA for 1000 hrs (from [34]). Figures from [7].

The map of the surface brightness at $2.675 \mathrm{GHz}$ produced according to these models is reported in Fig.4 (left panel). The distribution of DM in Coma gives origin to a radio emission at this frequency with a shape that is quite similar to the observed one. We also note that the procedure 
of obtaining the maps of diffuse radio emission is quite delicate, and depends critically on the removal of point or slightly extended sources, and can alter the real shape of the diffuse emission (for example, note that the radio halo maps observed at 1.4 and $2.675 \mathrm{GHz}$ have peaks in different positions $[24,28]$ ), because by subtracting the emission of discrete sources it is possible that also part of the DM emission in the same location is subtracted, as already noted in the Bullet cluster.
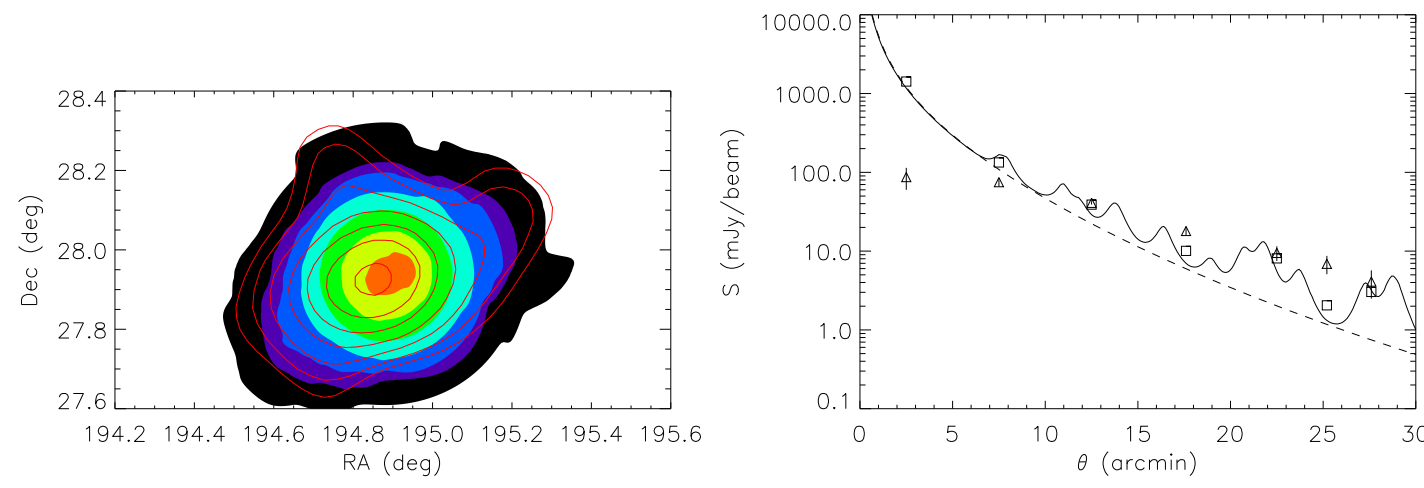

Figure 4: Left Panel: map of the radio surface brightness at $2.675 \mathrm{GHz}$ in Coma from the DM halos for the $9 \mathrm{GeV}$ neutralino model as in Fig.3. The map is smoothed on a scale of 9.35 arcmin, and is compared with the contours of fig.4 of Thierbach et al. [28]. Right Panel: azimuthally averaged radio surface brightness at $1.4 \mathrm{GHz}$ for the same $9 \mathrm{GeV}$ neutralino model as in left panel. The solid line is the total emission, and the dashed line is the emission of the main halo. Triangles are data from Deiss et al. [24], squares are the average of the surface brightness calculated in concentric rings with the same centers and extensions than in Deiss et al. [24]. Figures from [7].

The azimuthally averaged radio surface brightness profile at $1.4 \mathrm{GHz}$ produced in this model is shown in Fig.4 (right panel), compared with the data of Deiss et al. [24]. In the same figure we also show with the squares the average of the radio surface brightness calculated in concentric rings having the same properties than in Deiss et al. [24]. We notice that, outside the most internal circle having a radius of $\sim 5 \operatorname{arcmin}$ (where the radio emission is probably dominated by the halo around the cD galaxy NGC 4874), the DM sub-halos produce an average surface brightness profile wider than the emission of the main DM halo alone, allowing to have a better agreement with the observed data out to large radii, that instead is not possible to obtain by considering the main DM halo alone.

\section{Discussion and conclusions}

The combination of radio and gravitational lensing observations of galaxy clusters is a very promising way to obtain information about the physics of galaxy clusters and the properties of DM. The spatial information about the DM distribution, combined with information on the baryonic matter derived from X-rays, can allow to identify the regions where the DM is expected to be dominant, and attribute the radio emission produced in these regions to the electrons produced by DM annihilation. In this paper we have presented two examples of application of this technique. 
In the Bullet cluster the spatial displacement of the regions where the baryonic and the DM are dominant allows to study the radio spectrum in the different regions using different models. At the moment the lack in literature of separated spectral data for the BS and the DME regions does not allow to obtain definite conclusions; a contribution from the DM to the overall radio emission is possible because of the spectral changes visible in the total spectrum, that suggest that more than one component is producing the diffuse radio emission; possibly even some compact or slightly extended sources, if located far from visible galaxies and close to DM peaks, can be due to DM annihilation.

In the Coma cluster the distribution of the DM sub-halos closely resembles the radio halo surface brightness shape, and the model with mass $9 \mathrm{GeV}$ and composition $\tau^{+} \tau^{-}$allows to reproduce well the overall spectrum for the observed value of the magnetic field without violating the gamma ray upper limits. An open issue is related to the values of the DM annihilation cross section and the substructures boosting factor: if the value of the cross section is of the order of the one found in a study of the Galactic Center gamma ray excess [27], the required value of the boosting factor is of the order of what is expected in galaxy clusters, whereas if the value of the cross section is lower as suggested in other studies [30,31], the required value of the boosting factor is higher. However, other baryonic sources of diffuse radio emission can be present in the cluster, like secondary electrons of hadronic origin, electrons accelerated or re-accelerated by shocks or turbolences, electrons emitted by radio galaxies lobes (see, e.g. [2]), and therefore it is possible that the DM-produced electrons produce only a fraction of the total emission; in this case the required value of the product of the annihilation cross section by the substructures boosting factor would be smaller.

We also checked the dependence of these results on the assumptions that have been made to describe the properties of DM halos. One possible difference compared to the model for the profile of DM density as described in Sect.2 is the use of cored radial profiles having a flatter shape in the internal part of the halo, as possibly found in halos of size of galaxies or smaller (e.g. [35]). In this respect, we note that the radius of the largest sub-halo in the Coma cluster is of the order of 115 $\mathrm{kpc}$, corresponding to $\sim 4$ arcmin at the distance of Coma; since we are comparing our results with surface brigthness profiles having HPBW of 9.35 arcmin [24], we expect that a different shape of the internal profile of sub-halos should be diluted inside the beam, and the difference should not impact heavily on the contribution of sub-halos to the surface britghntess profile of the whole cluster. A different conclusion should be obtained if also the main halo should have a cored profile: in this case we would expect that the central cusp in the surface brightness profile should be less evident, and the accordance of this profile with the observed one might be more accurate in the central region of the cluster compared with the results presented in this paper (see Fig.4).

Another possible issue is the correct relation between the halo mass and the concentration parameter (eq.2.2), that in recent papers has been recalculated on the basis of results of recent numerical simulations (e.g. [36]). Using the mass/concentration relation as presented in the last cited paper, we calculated the radio flux produced by DM annihilation for halos located at the redshift of Coma, with different values of the mass between $10^{12}$ and $10^{15} \mathrm{M}_{\odot}$, and for a fixed magnetic field of $3 \mu \mathrm{G}$. We have found that the flux expected from a generic halo obtained using the new concentration model is smaller than the one found with our model, and the difference is bigger for small sized halos. Specifically, the radio flux produced by halos with mass $10^{12}-10^{13} \mathrm{M}_{\odot}$ with this assumption is of the order of 3.3-2.4 times smaller than the one found in our results, 
whereas for $10^{15} \mathrm{M}_{\odot}$ the flux is basically the same. This result would imply a smaller contribution of sub-halos in the Coma cluster, that would reflect both in the estimation of the necessary cross section (but not heavily, because most of the contribution comes from the main halo), and in the surface brightness profile of the whole cluster (where the sub-halos contribution would become less evident). Therefore more accurate calculations, taking into account these possible differences in the DM profiles, would be desirable in order to estimate the strength of the results presented in this paper.

On the basis of these results, the DM origin for part of the diffuse radio emission in galaxy clusters is a possibility that deserves to be better explored and constrained through the combination of radio and gravitational lensing measures, together with measures in other spectral bands like Xrays and gamma rays. The present results suggest that a contribution from DM can be important, but require to study in more detail the spectrum of the diffuse radio emission in smaller regions of the cluster in order to identify in which regions the contribution from DM can be relevant. The extension of this technique to other clusters, as well the study of the statistical properties of clusters at the light of DM models, will be important. In this respect, we note that the properties of the DM suggested by the studies in the Bullet and the Coma cluster are different: in the Bullet cluster the best possibility is given by a neutralino with mass $500 \mathrm{GeV}$ and composition $W^{+} W^{-}$, whereas in the Coma cluster the best fit is given by a neutralino with mass $9 \mathrm{GeV}$ and composition $\tau^{+} \tau^{-}$. Since the magnetic field properties are better constrained in the Coma cluster, the second possibility at the moment appears to be favoured, but new studies in other clusters where radio and gravitational lensing measures are available, like the clusters A520 (Marchegiani et al., in preparation) and A1682 (Khanye et al., in preparation), are desirable to obtain better information.

\section{Acknowledgements}

This work is based on the research supported by the South African Research Chairs Initiative of the Department of Science and Technology and National Research Foundation of South Africa (Grant No 77948). PM acknowledges support from the Department of Science and Technology/National Research Foundation (DST/NRF) Square Kilometre Array (SKA) post-graduate bursary initiative under the same Grant.

\section{References}

[1] Colafrancesco S., Profumo S., Ullio P., 2006, A\&A, 455, 21

[2] Feretti L., Giovannini G., Govoni F., Murgia M., 2012, A\&ARv, 20, 54

[3] Colafrancesco S., Lieu R., Marchegiani P., Pato M., Pieri L., Buonanno R., 2011, A\&A, 527, A80

[4] Okabe N., Futamase T., Kajisawa M., Kuroshima R., 2014, ApJ, 784, 90

[5] Clowe D., et al., 2006, ApJ, 648, L109

[6] Marchegiani P., Colafrancesco S., 2015, MNRAS, 452, 1328

[7] Marchegiani P., Colafrancesco S., 2016, JCAP, 11, 033

[8] Bullock J.S. et al., 2001, MNRAS, 321, 559 
[9] Colafrancesco S., Marchegiani P., Beck G., 2015, JCAP, 02, 032

[10] Coe D., 2010, arXiv:1005.0411

[11] Gondolo P., Edsjo J., Ullio P., Bergstrom L., Schelke M., Baltz E.A., 2004, JCAP, 07, 008

[12] Pieri L., Lavalle J., Bertone G., Branchini E., 2011, Phys. Rev. D., 83, 023518

[13] Bradac M., et al., 2006, ApJ, 652, 937

[14] Shimwell T.W., et al., 2014, MNRAS, 440, 2901

[15] Ota N., Mitsuda K., 2004, A\&A, 428, 757

[16] Wik D.R., et al., 2014, ApJ, 792, 48

[17] Markevitch M., et al., 2002, ApJ, 567, L27

[18] Liang H., Hunstead R.W., Birkinshaw M., Andreani P., 2000, ApJ, 544, 686

[19] Colafrancesco S., Marchegiani P., 2008, A\&A, 484, 51

[20] Colafrancesco S., Marchegiani P., Buonanno R., 2011, A\&A, 527, L1

[21] Ackermann M., et al., 2014, ApJ, 787, 18

[22] Barrena R. et al., 2002, A\&A, 386, 816

[23] Brown S., Rudnick L., 2011, MNRAS, 412, 2

[24] Deiss B.M., Reich W., Lesch H., Wielebinski R., 1997, A\&A, 321, 55

[25] Bonafede A., et al., 2010, A\&A, 513, A30

[26] Briel U.G., Henry J.P., Boehringer H., 1992, A\&A, 259, L31

[27] Abazajian K.N., Keeley R.E., 2016, Phys. Rev. D, 93, 083514

[28] Thierbach M., Klein U., Wielebinski R. 2003, A\&A, 397, 53

[29] Ishiyama T., 2014, ApJ, 788, 27

[30] Ackermann M., et al., 2015, Physical Review Letters, 115, 231301

[31] Ade P.A.R., et al., 2016, A\&A, 594, A13

[32] Beck G., Colafrancesco S., 2016, JCAP, 05, 013

[33] Vazza F., et al., 2016, MNRAS, 459, 70

[34] Funk S., Hinton J.A., 2013, Astroparticle Physics, 43, 348

[35] McGaugh S.S., de Blok W.J.G., 1998, ApJ, 499, 41

[36] Prada F., Klypin A.A., Cuesta A.J., Betancort-Rijo J.E., Primack J., 2012, MNRAS, 423, 3018 